1

\title{
Understanding the mechanical behaviour of fibre/matrix interfaces during push-in tests by means of finite element simulations and a cohesive zone model
}

\author{
D. Esqué-de los Ojos ${ }^{1 *}$, R.Ghisleni², A. Battisti ${ }^{3 \dagger}$, G. Mohanty ${ }^{4}$, J. \\ Michler $^{4}$, J. Sort ${ }^{5}$, A.J. Brunner ${ }^{3}$ \\ ${ }^{1}$ Departament de Física, Universitat Autònoma de Barcelona, E-08193 Bellaterra, Spain \\ ${ }^{2}$ Laboratory for Advanced Materials Processing, \\ EMPA Materials Science \& Technology, CH-3602 Thun, Switzerland \\ ${ }^{3}$ Laboratory for Mechanical Systems Engineering, \\ EMPA Materials Science \& Technology, CH-8600 Duebendorf, Switzerland \\ ${ }^{4}$ Laboratory for Mechanics of Materials and Nanostructures, \\ EMPA Materials Science \& Technology, CH-3602 Thun, Switzerland \\ ${ }^{5}$ Institució Catalana de Recerca i Estudis Avançats (ICREA) and Departmanet de Física, \\ Universitat Autònoma de Barcelona, E-08193 Bellaterra, Spain
}

\begin{abstract}
The present work represents a progress towards the understanding of the mechanical behaviour of the fibre/matrix interface during push-in tests of fibre-reinforced polymer-matrix composites. Finite element simulations incorporating a cohesive zone model are used for this purpose. Different values of interface strength, interface fracture toughness, fibre diameter and friction coefficient are considered to study how they affect the load-displacement curves. A critical value of the displacement exists, being independent of the fibre diameter for given values of interface strength and fracture toughness, marking the separation between two regimes: (i) a cohesive-dominated zone interaction and (ii) a frictional contact between debonded fibre and matrix. Maps showing the different regimes are constructed, proving their helpfulness to tune the mechanical properties of the interface in order to favour a certain mechanical response. Finally, we study the debonding velocity and how this is affected by the mechanical properties of the interface providing an empirical relation.
\end{abstract}

\section{Submitted to Computational Materials Science}

February 5, 2016

Preprint of: Esqué-de los Ojos, D. et al. "Understanding the mechanical behaviour or fiber/matrix interfaces during push-in tests by means of finite element simulations and a cohesive zone model" in Computatonal materials science (Ed. Elsevier), vol. 117 (May 2016), p. 330-337. The final version is available at DOI 10.1016/j.commatsci.2016.02.009

*Corresponding author. e-mail: danielesque@gmail.com. Present address: School of Materials, The University of Manchester, M13 9PL, UK

${ }^{\dagger}$ Present address: Omya International AG, CH-4665 Oftringen, Switzerland 


\section{Introduction}

Composite materials that combine the properties of at least two single-phase materials in a synergistic manner have been widely investigated over the years in order to enhance the properties or to create new functionalities that are not attainable using the individual constituent materials separately [1]. Basically, in fibre-reinforced materials, the matrix transfers the stress to the fibres, which, due to their higher load resistance, enhances the amount of stress that the composite can bear before failure. This stress transfer will occur along the fibre/matrix interface and in consequence, will depend on the bounding between the fibre and the matrix or, in other words, on the mechanical properties of the in-terface. Hence, it is easy to understand the great deal of interest in the field of interface tailoring. The reader is encouraged to consult the recent work from Karger-Kocsis et al. [2] where a thorough review, since the year 2000, on the recent advances in fibre/matrix interface engineering can be found. Among other things, different fibre types (organic and inorganic) along with routes for interface treatment are presented. Some examples are the sizing of glass to protect them from fracture and of carbon fibres to improve interfacial fracture toughness when using polyaryletherketones (a type of thermoplastic polymer). An alternative route presented in [2] is the addition of nano fillers into sizing formulations, having the advantage of enhancing the roughness of the fibre, increasing the local modulus of the fibre and hence the shear strength and, finally, allowing the possible structuring of nano fillers for sensing applications. As carbon fibres are one of the most studied reinforcing structures, it is also interesting to mention the work by Sharma et al. [3] reviewing the topic of the surface modification of carbon fibres and also the carbon fibre/polymer interfacial adhesion.

As aforementioned, the mechanical performance of composite materials depends not only on the mechanical properties of each individual phase or component but also on the interactions between them. For this reason, the study of the mechanical performance of fibre-reinforced composites is an active topic, especially regarding the study of the fibre/matrix interface [4] - [9]. Some previous works have emphasized especially the optimization of the interface to attain optimal performance [10] - [12]. A good understanding of the mechanical behaviour of the fibre/matrix interface is needed in order to identify the most critical parameters governing the mechanial performance of this type of systems.

The typical experiment to test fibre-reinforced composites is the push-in or push-out of a single fibre with a cylindrical flat punch whose diameter is smaller than that of the fibre. 
In the literature one can also find some examples showing the study of interfacial properties in fibre-reinforced composites by means of finite element (FE) simulations. Chandra and Ghonem [12] conducted simulations of push-out tests on titanium matrix composites at room and elevated temperatures where silicon carbide fibres were chosen as the reinforcing material. In their work, they introduced a novel FE analysis to extract values of shear and frictional strength along with the fracture energy values associated with the interfaces. In doing so, they compared different interface models, being one of them the cohesive zone model that consists of a constitutive relation between the tractions acting on the interface and the corresponding interfacial separation (debonding). This cohesive zone formulation is based on the works by Barenblatt and Needleman [14],[15] along with constitutive relations presented by Lin at al. [16]. Lin et al. [17] also used the cohesive zone model in a parametric study of the effects of the friction coefficient, the interfacial bond strength and the process-induced residual stresses on the fibre/matrix interface failure system. They showed that their model was able to capture the behaviour of a polyester/epoxy composite during push-out tests. Chen et al. [18] presented FE simulations studying the interface behaviour of $\mathrm{Al}_{2} \mathrm{O}_{3}$ fibre-reinforced $\mathrm{NiAl}$ composites at room and high temperatures. The objective was to understand the interfacial behaviour of that kind of intermetallic matrix composites and to correlate the mechanical performance of the interface with experimental data to extract the intrinsic shear strength of the interface. Also, they reached the conclusion that fibre debonding can occur at the top side for thicker samples and at the bottom side for thinner samples. You et al. [19] used the cohesive zone model with a linear elastic traction-separation law to investigate the push-out behaviour of SiC fibre-reinforced copper matrix composites. In doing that, they found excellent agreement between simulations and experiments, using an inverse fitting process to calibrate the tractionseparation law and the damage evolution law. Finally, Jäger et al. [8] used the same cohesive zone model as in [5] and [6] to assess the behaviour of the fibre/matrix interface using an energy-based evaluation of the interfacial failure

In a previous publication [6], the authors performed single fibre push-out tests on carbonfibre epoxy laminates with different fibre treatment (comparing desized fibres as reference with fibres with deposited, COOH-functionalized carbon nanotubes and with fibres oxidized by the application of electric fields in a water-based suspension) using thin laminate slices (roughly 30 micron thick). They further, applied the energy analysis approach proposed for $\mathrm{SiC}$ ceramic matrix composites by Mueller et al. [9] to their systems and complemented 


\section{Finite element simulations and the cohesive zone model}

Finite element (FE) simulations were performed to study the behaviour of the interface between carbon fibres and an epoxy polymeric matrix, in which the fibres were embedded, under fibre push-in test test. In doing so, four parameters were taken into account: diameter of the fibres $(D)$, interface strength $(\tau)$, interface fracture toughness $(\Gamma)$ and friction coefficient $(\mu)$ between the fibre and the matrix. The values considered for each of the aforementioned parameters were: $D=4,5,7$ and $9 \mu \mathrm{m} ; \tau=50,75,100,200,300$ and $500 \mathrm{MPa} ; \Gamma=100$, 150,200 and $250 \mathrm{~J} / \mathrm{m}^{2}$ and $\mu=0$ and 0.05 . Combination of all these values gave as a result almost 200 simulations. The bearing-in-mind idea was to study how these four parameters were affecting the response of the fibre/matrix interface during push-in of an isolated fibre. The mesh used for the simulations consisted of 27,760 - 48,020 (depending on the diameter of the fibre) 4-node fully- integrated elements. Push-in modelling of a single fibre in a matrix was considered to be axisymmetric. Length of the fibres was kept constant and equal to 375 
$\mu \mathrm{m}$. Figure 1 shows a sketch of the finite element model. The fibre/matrix interface was considered as infinitely thin. During preparation of the model, a cohesive fibre/matrix zone interaction [5], [6], [8], [13], relating stress and displacements at the interface (Eqs. 1 and 2), was assumed along with a quadratic failure criterion (Eq. 3) governing the initiation of damage. Once damage was initiated at the interface, contact between fibre and matrix was ruled by Coulomb friction, with a friction coefficient $\mu$. It is worthy to mention here that the considered values for $\mu=0$ and 0.05 were arbitrary and had the sole purpose of comparing a frictionless and friction contacts between the debonded fibre and the matrix.

$$
\begin{aligned}
& t=\sqrt{\left\langle t_{\mathrm{n}}\right\rangle^{2}+t_{\mathrm{t}}^{2}+t_{\mathrm{s}}^{2}} \\
& \delta=\sqrt{\delta_{\mathrm{n}}^{2}+\delta_{\mathrm{t}}^{2}+\delta_{\mathrm{s}}^{2}}
\end{aligned}
$$

where $t$ is the total stress at the interface and $\delta$ the total displacement. Sub-index $n$ refers to normal to the surface while $t$ and $s$ refer to the transversal directions. Brackets for $t_{\mathrm{n}}$ in Eqs. 1 and 3 denote the Macaulay brackets, that return the argument if positive and give a zero if negative. This means that only tensile stresses are taken into account during the calculations.

$$
\left(\frac{\left\langle t_{\mathrm{n}}\right\rangle}{t_{\mathrm{n}}^{c}}\right)^{2}+\left(\frac{t_{\mathrm{t}}}{t_{\mathrm{t}}^{c}}\right)^{2}+\left(\frac{t_{\mathrm{s}}}{t_{\mathrm{t}}^{c}}\right)^{2}=1 .
$$

Here, $t_{\mathrm{t}}^{c}=t_{\mathrm{s}}^{c}$ as it was considered that both transversal directions are equivalent. Additional details on the constitutive equations can be found elsewhere [5], [6].

For the different parts of the model, we considered the polymeric matrix to behave as a purely elastic material, the flat punch exerting the load during the push-in test as a perfectly rigid body with a frictionless contact with the top part of the fibre. As for the fibre, we considered it as a transversely isotropic material. Additionally to the aforementioned simulations, the Supplementary Material of the electronic version of the present paper shows results concerning: (i) the effect of the matrix's Young's modulus on the push-in load - displacement curves and (ii) the influence of thermal stresses on the push-in load - displacement curves and also on the damage (debonding) at the fibre/matrix interface. It is important to take into account thermal stresses in the model, as during curing and annealing processes one can reach differences in temperature of $100 \mathrm{~K}$, inducing the onset of debonding prior to the push-in test. Effect of residual stresses during pull-out was already explored by Bheemreddy et al. [20] 


\section{Results and discussion}

\subsection{Fiber push-in simulations: Applied load - top fiber displacement analysis}

Figure 2 shows the load - displacement curves for different values of $\Gamma$ and $\tau, P$ and $d$ being the applied load by and the displacement of the punch pushing the fibre, respectively. Each part of Fig. $2((\mathrm{a})$ to $(\mathrm{h}))$ shows results for different diameters of the fibre $(D)$ and friction coefficients 

Here, just a small selection of the simulated $P-d$ curves is presented, the additional curves can be found in the Supplementary Material available in the on-line version of the present article. Comparing parts with the same value of $\Gamma$, one can see that an increase in $\tau$ increases the elastic regime, characterized by a linear dependence between $P$ and $d$, during the push-in of the fibre. Interestingly, one can do an analogy between these curves and the uniaxial stress - strain behaviour during the tensile test of a material. The interfaces characterized by a low value of interface strength $\tau$ (left column with $\tau=50 \mathrm{MPa}$ ) follow a power-law relation, that saturates for those cases with $\mu=0$. Instead, interfaces with higher values of $\tau$ (right column, with $\tau=500 \mathrm{MPa}$ ) resemble an elastic-perfectly plastic material (with saturation for $\mu=0$ and linear increase of load for $\mu=0.05$ ). This is a worthy comparison as it indicates that by increasing the value of $\tau$, the interface becomes more rigid i.e., higher loads will be needed to reach the point at which the interface behaviour is dominated by the friction between the matrix and the debonded part of the fibre. The point at which this change in regime occurs can be easily identified in the different parts of Fig. 2 (and Figs. S2 - S5 in the Supplementary Material) as it corresponds to the displacement $d$ where the load saturates for $\mu=0$. We designate the displacement at which $P$ becomes linear with $d$ (saturated for $\mu=0$ and monotonically increasing for $\mu=0.05)$ as the critical punch displacement $d_{\mathrm{cr}}$. This displacement sets the boundary between two different behaviours: a cohesive-interaction regime for displacements below $d_{\mathrm{cr}}$ and a frictional regime for displacements above $d_{\mathrm{cr}}$. Figure 3 shows additional simulations for combinations of mechanical parameters that need $d \geq 6 \mu \mathrm{m}$ to saturate P. Remarkably, from Fig. 2 (and Figs. S2 - S5 in the Supplementary Material) it can be seen that, for a given combination of interface properties, $d_{\mathrm{cr}}$ is independent of the diameter of the fibre. Note that simulations in Fig. 3 are only for a unique value of the fibre diameter, $D=4 \mu \mathrm{m}$. It is also worthy to mention from Fig. 2(a) that load slightly increases for $\mu=0$ and $D=9 \mu \mathrm{m}$. The reason for this increase in load is that the fibre has debonded completely and, since the bottom part of the fibre in our model is tight to prevent vertical displacement of the entire fibre (in other words, the model considers a pushin simulation, but not a push-out of the fibre), the load starts to increase as a consequence of the inability of the fibre to slide out. In order to account for the effects of thermal stresses, e.g., from the curing process, FEM simulations were performed comparing systems with and without thermal stresses (see Figure S6 in the Supplementary Material for a selection of these 


\subsection{Active deformation regime during fiber push-in: cohesive interaction vs. frictional contact}

By collecting the values of $d_{\mathrm{cr}}$ for each pair of $\tau$ and $\Gamma$ we can build-up a deformation map showing the governing regime during push-in. Figure 4 represents a $\tau-d_{\text {cr }}$ plot for different $\Gamma$ s and, analogously, Figure 5 shows the $\Gamma-d_{\text {cr }}$ plot for different values of $\tau$. In Fig. 4 , it can be seen how each series of data for a given value of $\Gamma$ delimits the range of displacements that will be governed by the cohesive interaction (left-hand side) and those affected by the frictional contact between the part of debonded fibre and the matrix (right-hand side). Also in Fig. 4, it can be seen that, by increasing the value of $\tau$, the range of variation of $d_{\mathrm{cr}}$ values is reduced, from $\sim 4 \mu \mathrm{m}$ to $\sim 10 \mu \mathrm{m}$ at $\tau=50 \mathrm{MPa}$ and from $\sim 0.5 \mu \mathrm{m}$ to $\sim 1.5 \mu \mathrm{m}$ at $\tau=$ $500 \mathrm{MPa}$, when changing the value of $\Gamma$. In other words, increasing the value of the interface strength $\tau$ reduces the variability of the critical punch's displacement delimiting the transition between both regimes occurring at different values of the interface fracture toughness. For lower values of $\tau$, changes in $\Gamma$ imply a great variation on the values of $d_{\mathrm{cr}}$. In turn, for a given $\Gamma$, only if $\Gamma$ is high a change in $\tau$ results in an appreciable change of $d_{\mathrm{cr}}$. Fig. 4 is useful to predict whether it makes sense or not, for a given value of $\tau$, to vary the value of $\Gamma$ in order to promote a certain deformation regime (i.e., dominated by fibre debonding or by frictional contact). From an experimental point of view, changes in the deformation regime, most likely require changing either the fibre or the matrix material. Indeed, Battisti et al. [6] showed that surface modification by means of electrophoretic deposition of carbon nanotubes (CNT) or oxidation of the surface lead to similar debonding initiation, i.e., none or little effect of treatment on interfacial adhesion, but significantly higher loads were required to push-out the fibres when using CNTs (see Fig. 8 (a) in [6] for details). 
Figure 5 reveals that the evolution of the punch's critical displacement with the interface fracture strength is linear. Analogous to Fig. 4, for each value of interface strength, displacements at the left-hand side of the lines indicate cohesive-interaction dominated regimes, while displacements at the right-hand side of the lines indicate the regime dominated by the frictional behaviour between the fibre and the matrix. Again, for a given $\tau$, the greatest variation of $d_{\mathrm{cr}}$ is obtained for higher values of $\Gamma$. In turn, for a given value of $\Gamma$, interfaces with lower values of $\tau$ will be more sensitive to changes of $\Gamma$. From Fig. 4 we can see that, for the limiting case of $\tau \rightarrow \infty$, changing the value $\Gamma$ will not be translated into a change of $d_{\mathrm{cr}}$. Notice here that both Figs. 4 and 5 do not depend on the friction level between the debonded fibre and the matrix, in agreement with Fig. 2 where it is shown that the $d_{\mathrm{cr}}$ is independent of both $\mu$ and $D$. As shown in Fig. S6, $d_{\text {cr }}$ does not depend on having or not thermal stresses and concomitantly, thermal stresses neither affect Figs. 4 and 5. However, Figure S8 shows the initial debonding for two different fibres' diameters and different interfacial strengths. This initial debonding is caused by the differences on the thermal coefficient expansions of the fibre and the matrix. The initial debonding depends on the fibre's diameter, being higher for bigger fibres, but as the interfacial strength increases this difference is reduced and, eventually, for high enough values of $\tau$, this initial debonding difference disappears. Here it is worthy to remark that present simulations correspond to push-in experiments on isolated fibers. One would expect that, when taking into account the effect of surrounding fibers, the tendency would be to have stiffer structures and hence, that curves in Figs. 4 and 5 could be displaced to the right. However, a recent investigation (to be published) performed by the same authors show that the initiation of fiber debonding is independent of both fiber treatment and fiber packaging, analogous to [6], but for another fibre type and a thicker slice that results in fibrepush-in rather than push-out. In order to accurately study the effect of neighbouring fibers a three-dimensional mesh would be needed.

\subsection{Speed of crack propagation in terms of interfatial mechanical properties}

Another interesting output of the present analysis is the study of the crack length propagated across the fibre/matrix interface as a function of the punch's displacement. To establish the crack length one can track, during the simulations, which nodes at the interface fulfill the quadratic failure criterion (Eq. 3). Knowing the internode separation and how many nodes have failed in each simulation one can infer the crack length. Figure 6 shows one example 
where $a \equiv a(\tau)$ and $b \equiv b(\tau)$. This fitting process was done for the different values of $\tau$ at constant $\Gamma$ and $\mu$. Table 3 summarizes the values for the coefficients $a$ and $b$. It should be noted that for $\tau \leq 100 \mathrm{MPa}$, the $m$ vs $D$ curves tend to overlap. For each value of $\Gamma$ and $\mu$, the different values of $a$ and $b$ obtained from the fitting can, in turn, be fitted using the following set of equations

$$
\begin{gathered}
a=a_{1}-\left(a_{1}-a_{2}\right) \times e^{-\left[\left(a_{3} \tau\right)^{a_{4}}\right]} \\
b=b_{1}-\left(b_{1}-b_{2}\right) \times e^{-\left[\left(b_{3} \tau\right)^{b_{4}}\right]} .
\end{gathered}
$$

Table 4 summarizes the coefficients $a_{\mathrm{i}}$ and $b_{\mathrm{i}}$ for $\mu=0$ and $\mu=0.05$. Eqs. 5 and 6 along with coefficients given in Table 4 proved to be useful to see how, for a given interface, changes on $\tau, \Gamma$ and/or $\mu$ affect the velocity of crack propagation at the fibre/matrix interface. In spite of the relatively complex expressions defining $a$ and $b$ coefficients, some qualitative conclusions can be drawn. From Table 3 one can see that for small values of $\Gamma$, parameters $a$ and $b$ remain almost constant for any value of $\tau$, meaning that the slope $m$, or how fast the fibre is debonding, does not depend on the value of the interface strength. Even for larger $\Gamma$ values, $m$ does not significantly vary with $\tau$ for $\tau \geq 200 \mathrm{MPa}$. In other words, even for high $\Gamma$, a critical value of $\tau$ will be reached beyond which the debonding speed is not affected. This result goes on the same lines as what has been discussed for Figs. 4 and 5. In Fig. 4 it was shown that, for high values of $\tau$, variations in $\Gamma$ had almost no effect on the critical punch displacement delimiting the cohesive-interaction and frictional regimes. Analogous, for a given value of $\Gamma$, just when this value was high, a variation of $\tau$ translated into a significant change of $d_{\mathrm{cr}}$. Fig. 5 corroborated the aforementioned results, i.e., for high values of $\tau$, the variation of $\Gamma$ was not affecting the value of $d_{\mathrm{cr}}$. So, what transpires from all this discussion is that a contour exists 
in the range of the interface properties to favour a certain regime (cohesive vs. friction) or to modify the debonding speed between the fibre and the matrix.

\section{Conclusions}

This works presents finite element simulations on the push-in of fibre-reinforced composites by recourse of a cohesive zone model, inspired by experimental push-out and push-in tests. The study has taken into account different values for the diameter of the fibres, interface strength, interface fracture toughness and friction coefficient between the fibre and the matrix. In doing so, the existence of a critical displacement of the top part of the fibre has been shown. This critical displacement separates the load - displacement curves obtained during push-in experiments into two different regimes: one governed by the cohesive interaction between the fibre and the matrix, and another ruled by the frictional contact between the debonded part of the fibre and the matrix. By plotting the critical displacement against the mechanical properties of the fibre/matrix interface, the existence of a limiting value for the interface strength was unveiled, above which no significant variation on the critical displacement value could be achieved. Finally, empirical relations for the speed of fibre debonding are provided, showing, again, that a limiting value for the interfacial strength exists, beyond which the debonding speed is no longer affected. Our results provide valuable information in order to predict the deformation behaviour of the fibre-reinforced composite materials and, in particular, to decide whether further change in the interfacial properties of the fibre/matrix system could allow an enhancement of the composite mechanical endurance.

\section{$5 \quad$ Acknowledgments}

Andrea Battisti acknowledges partial funding by the Commission for Technology and Innovation (CTI Project Nr. 11237.1 PFIW-IW) of the Swiss Confederation and a COFUND Marie Curie project. Jordi Sort acknowledges financial support from 2014-SGR-1015 from D.G.U. Catalunya.

\section{References}

[1] P.K. Mallick. Fiber-reinforced materials: Materials, Manufacturing, and Design. CRC Press FL, USA. Taylor and Francis, 2007. 
[2] J. Karger-Kocsis, H. Mahmood, A. Pegoretti. Progress Mater. Sci. 73 (2015) 1.

[3] M. Sharma, S. Gao, E. Mäder, H. Sharma, L.Y. Wei, J. Bijwe. Compos. Sci. Technol. 102 (2014) 35 .

[4] A. Pegoretti, M. Fidanza, C. Migliaresi, A.T. DiBenedetto. Compos. Part A 29 (1998) 283.

[5] M. Rodriguez, J.M. Molina-Aldareguia, C. Gonzalez, J. Llorca. Compos. Sci. Technol. 72 (2012) 1924.

[6] A. Battisti, D. Esqué-de los Ojos, R. Ghisleni, A.J. Brunner. Compos. Sci. Technol. 95 (2014) 121.

[7] M. Greisel, J. Jäger, J. Moosburger-Wil, M.G.R. Sause, W.M. Mueller, S. Horn.Compos. Part A 66 (2014) 117.

[8] J. Jäger, M.G.R. Sause, F. Burkert, J. Moosburger-Wil, M. Greisel, S. Horn. Compos. Part A 71 (2015) 157.

[9] W.M. Mueller, J. Moosburger-Will, M.G.R. Sause, S. Horn. J. Eur. Ceram. Soc. 33 (2013) 441.

[10] F.R. Jones. J. Adhes. Sci. Technol. 24 (2010) 171.

[11] A. Orifici, I. Herszberg, R. Thomson. Compos. Struct. 86 (2008) 194.

[12] Jr. L. Mishnaevsky, P. Brøndsted. Comput. Mater. Sci. 44 (2009) 1351.

[13] N. Chandra, H. Ghonem. Compos. Part A 32 (2001) 575.

[14] G.I. Barenblatt. Adv. Appl. Mech. 7 (1962) 55.

[15] A. Needleman. J. Appl. Mech. 54 (1987) 525.

[16] G. Lin, A. Cornec, K.H. Schwalbe. Fatigue Fract. Eng. Mater. Struct. 21 (1998) 1159.

[17] G. Lin, P.H. Geubelle, N.R. Sottos. Int. J Solids Struct. 38 (2001) 8547.

[18] H. Chen, W. Hu, Y. Zhong, G. Gottstein. Mater Sci Eng 460 (2007) 624.

[19] J.H. You, W. Lutz, H. Gerger, A. Siddiq, A. Brendel, C. Höschen, S. Schmauder. Int. J. Solids Struct. 46 (2009) 4277. 
[20] V. Bheemreddy, K. Chandrashekhara, L.R. Dharani, G.E. Hilmas. Comput. Mater. Sci. 79 (2013) 663.

[21] Unpublished results from EMPA Materials Science \& Technology

[22] V. Ganesh, N. Naik. Compos. Sci. Technol. 51 (1994) 387.

[23] F.N. Cogswell, Thermoplastic aromatic polymer composites. Chapter 2 Components of a thermoplastic structural composite, pp. 11-50, Butterworth-Heinemann, Oxford, United Kingdom, 1992.

[24] A. Vital, B. Doleman, M. Saad, Thermophysical Properties of AS4/3501-6 CarbonEpoxy Composite. Proceedings of the ASME 2013 International Mechanical Engineering Congress and Exposition IMECE2013-65186, November 15-21, 2013. San Diego, California, USA.

[25] Y. Jia, W. Yan, H-Y Liu. Comput. Mater. Sci. 62 (2012) 79. 


\section{Table 1}

Table 1. Mechanical ${ }^{(22)}$ and thermal ${ }^{(23)}$ properties for the fibre used during finite element simulations. Direction 1 goes along the fibre and directions 2 and 3 are transversal to the fibre.

\begin{tabular}{ccccccccc}
\hline \hline \multicolumn{1}{c}{ Fibre } \\
\hline $\begin{array}{c}E_{2}=E_{3} \\
(\mathrm{GPa})\end{array}$ & $E_{1}(\mathrm{GPa})$ & $v_{12}$ & $\begin{array}{c}\mathrm{G}_{13}=G_{23} \\
(\mathrm{GPa})\end{array}$ & $G_{12}(\mathrm{GPa})$ & $\begin{array}{c}\alpha_{2}=\alpha_{3} \\
\left(\mathrm{~K}^{-1}\right)\end{array}$ & $\begin{array}{c}\alpha_{1} \\
\left(\mathrm{~K}^{-1}\right)\end{array}$ & $\begin{array}{c}k_{2}=k_{3} \\
(\mathrm{~W} / \mathrm{m} \cdot \mathrm{K})\end{array}$ & $\begin{array}{c}k_{1} \\
(\mathrm{~W} / \mathrm{m} \cdot \mathrm{K})\end{array}$ \\
40 & 230 & 0.26 & 24 & 14.3 & $12 \cdot 10^{-6}$ & $-1.2 \cdot 10^{-6}$ & 3 & 16 \\
\hline \hline
\end{tabular}

\section{Table 2}

Table 2. Mechanical and thermal ${ }^{(24,25)}$ properties for the matrix used during finite element simulations.

\section{Matrix}

\begin{tabular}{cccc}
\hline$E(\mathrm{GPa})$ & $v$ & $\begin{array}{c}\alpha \\
\left(\mathrm{K}^{-1}\right)\end{array}$ & $\begin{array}{c}k_{1} \\
(\mathrm{~W} / \mathrm{m} \cdot \mathrm{K})\end{array}$ \\
300 & 0.35 & $62 \cdot 10^{-6}$ & 0.65 \\
\hline \hline
\end{tabular}




\section{Table 2}

Table 2. Parameters obtained for Eq. (4). Values in parenthesis correspond to $\mu=0.05$, while the others are for $\mu=0$.

\begin{tabular}{|c|c|c|c|c|c|c|c|c|}
\hline \multirow[b]{3}{*}{$\tau(\mathrm{MPa})$} & \multicolumn{8}{|c|}{$\Gamma$} \\
\hline & \multicolumn{2}{|c|}{$100 \mathrm{~J} / \mathrm{m}^{2}$} & \multicolumn{2}{|c|}{$150 \mathrm{~J} / \mathrm{m}^{2}$} & \multicolumn{2}{|c|}{$200 \mathrm{~J} / \mathrm{m}^{2}$} & \multicolumn{2}{|c|}{$250 \mathrm{~J} / \mathrm{m}^{2}$} \\
\hline & $a$ & $b$ & $a$ & $b$ & $a$ & $b$ & $a$ & $b$ \\
\hline 50 & $\begin{array}{c}22.07 \\
(14.46)\end{array}$ & $\begin{array}{c}3.55 \\
(3.32)\end{array}$ & $\begin{array}{c}19.66 \\
(13.48)\end{array}$ & $\begin{array}{c}2.95 \\
(2.96)\end{array}$ & $\begin{array}{c}18.66 \\
(13.13)\end{array}$ & $\begin{array}{c}2.80 \\
(2.80)\end{array}$ & $\begin{array}{c}16.91 \\
(12.91)\end{array}$ & $\begin{array}{c}2.95 \\
(2.72)\end{array}$ \\
\hline 75 & $\begin{array}{c}22.07 \\
(13.83)\end{array}$ & $\begin{array}{c}3.50 \\
(3.29)\end{array}$ & $\begin{array}{c}18.31 \\
(12.72)\end{array}$ & $\begin{array}{c}2.86 \\
(2.77)\end{array}$ & $\begin{array}{c}16.51 \\
(12.04)\end{array}$ & $\begin{array}{c}2.57 \\
(2.52)\end{array}$ & $\begin{array}{c}15.62 \\
(11.70)\end{array}$ & $\begin{array}{c}2.42 \\
(2.38)\end{array}$ \\
\hline 100 & $\begin{array}{c}22.06 \\
(13.64)\end{array}$ & $\begin{array}{c}3.45 \\
(3.27)\end{array}$ & $\begin{array}{c}18.10 \\
(12.39)\end{array}$ & $\begin{array}{c}2.83 \\
(2.73)\end{array}$ & $\begin{array}{c}15.93 \\
(11.56)\end{array}$ & $\begin{array}{c}2.49 \\
(2.42)\end{array}$ & $\begin{array}{c}14.65 \\
(11.07)\end{array}$ & $\begin{array}{c}2.28 \\
(2.23)\end{array}$ \\
\hline 200 & $\begin{array}{c}22.04 \\
(13.34)\end{array}$ & $\begin{array}{c}3.45 \\
(3.26)\end{array}$ & $\begin{array}{c}18.10 \\
(12.05)\end{array}$ & $\begin{array}{c}2.83 \\
(2.70)\end{array}$ & $\begin{array}{c}15.79 \\
(11.17)\end{array}$ & $\begin{array}{c}2.45 \\
(2.37)\end{array}$ & $\begin{array}{c}14.14 \\
(10.45)\end{array}$ & $\begin{array}{c}2.20 \\
(2.13)\end{array}$ \\
\hline 300 & $\begin{array}{c}22.04 \\
(13.20)\end{array}$ & $\begin{array}{c}3.46 \\
(3.25)\end{array}$ & $\begin{array}{c}18.10 \\
(11.89)\end{array}$ & $\begin{array}{c}2.82 \\
(2.71)\end{array}$ & $\begin{array}{c}15.76 \\
(11.00)\end{array}$ & $\begin{array}{c}2.45 \\
(2.37)\end{array}$ & $\begin{array}{c}14.17 \\
(10.35)\end{array}$ & $\begin{array}{c}2.19 \\
(2.13)\end{array}$ \\
\hline 500 & $\begin{array}{c}22.02 \\
(13.09)\end{array}$ & $\begin{array}{c}3.46 \\
(3.25)\end{array}$ & $\begin{array}{c}18.09 \\
(11.77)\end{array}$ & $\begin{array}{c}2.83 \\
(2.71)\end{array}$ & $\begin{array}{c}15.77 \\
(10.88)\end{array}$ & $\begin{array}{c}2.45 \\
(2.37)\end{array}$ & $\begin{array}{c}14.21 \\
(10.24)\end{array}$ & $\begin{array}{c}2.19 \\
(2.12)\end{array}$ \\
\hline
\end{tabular}

\section{Table 3}

Table 3. Parameters obtained for Eqs. 5 and 6 . Values in parenthesis correspond to $\mu=0.05$, while the others are for $\mu=0$.

\begin{tabular}{|c|c|c|c|c|c|c|c|c|}
\hline$\Gamma\left(\mathrm{J} / \mathrm{m}^{2}\right)$ & $a_{1}$ & $a_{2}$ & $a_{3}$ & $a_{4}$ & $b_{1}$ & $b_{2}$ & $b_{3}$ & $b_{4}$ \\
\hline \multirow{2}{*}{100} & 21.52 & 22.12 & 0.00 & 0.34 & 3.48 & 3.47 & 0.00 & -1.02 \\
\hline & (14.50) & (13.09) & (0.01) & $(-2.14)$ & (3.40) & (3.25) & $(0.03)$ & $(-1.86)$ \\
\hline \multirow{2}{*}{150} & 18.10 & 21.38 & 0.02 & 3.21 & 2.95 & 2.83 & 0.02 & -8.44 \\
\hline & (13.48) & (11.77) & (0.01) & $(-2.42)$ & (2.96) & (2.71) & $(0.02)$ & $(-6.25)$ \\
\hline \multirow{2}{*}{200} & 18.85 & 15.77 & 0.02 & -5.71 & 2.45 & 4.90 & 0.02 & -4.21 \\
\hline & (13.13) & (11.00) & (0.01) & $(-3.49)$ & (2.87) & (2.37) & $(0.02)$ & $(-4.22)$ \\
\hline \multirow{2}{*}{250} & 16.92 & 14.17 & 0.01 & -4.79 & 4.22 & 2.19 & 0.03 & -3.35 \\
\hline & $(14.30)$ & (10.24) & (0.02) & $(-2.19)$ & (2.82) & (2.12) & (0.02) & $(-3.53)$ \\
\hline
\end{tabular}




\section{Figure captions}

Figure 1. Schematic drawing showing the FE element model used in the present study. The fibre is considered as a transversely isotropic material while matrix is taken as an isotropic elastic solid. Interaction between fibre and matrix is modelled with a cohesive zone model and a frictional contact between the fibre and the matrix once debonding initiates, the fibre/matrix interface being infinitely thin. Vertical displacement of the bottom part of the system is prevented and, given the symmetry of the problem, here a two-dimensional axisymmetric mesh is used. Fibre is displaced downwards by a perfectly rigid flat punch with a diameter equal to the pushed fibre. Contact between the top-part of the fibre and the flat punch is considered frictionless in all cases.

Figure 2. Load $(P)$ - top fibre displacement $(d)$ for different combinations of interfacial strength $(\tau)$, interface fracture toughness $(\Gamma)$, friction coefficient $(\mu)$ and fibre diameter $(D)$. Closed symbols are for $\mu=0$ and open symbols for $\mu=0.05$. Left column is for $\tau=50 \mathrm{MPa}$ ((a) $\Gamma=100$ $\mathrm{J} / \mathrm{m}^{2}$; (c) $\Gamma=150 \mathrm{~J} / \mathrm{m}^{2}$; (e) $\Gamma=200 \mathrm{~J} / \mathrm{m}^{2}$; (g) $\Gamma=250 \mathrm{~J} / \mathrm{m}^{2}$ ) and right column for $\tau=500 \mathrm{MPa}$ ((b) $\Gamma=100 \mathrm{~J} / \mathrm{m}^{2}$; (d) $\Gamma=150 \mathrm{~J} / \mathrm{m}^{2}$; (f) $\Gamma=200 \mathrm{~J} / \mathrm{m}^{2}$; (h) $\Gamma=250 \mathrm{~J} / \mathrm{m}^{2}$ ). Vertical dotted lines indicate the value of the critical displacement $\left(d_{\mathrm{cr}}\right)$ marking the boundary between the two different interfacial behaviours (cohesive zone and frictional displacement) except for parts (c), (e) and (g), whose $d_{\mathrm{cr}}$ values are indicated in Fig. 3. Circled region in part (a) shows the increase in load for the case of $D=9 \mu \mathrm{m}, \tau=50 \mathrm{MPa}, \Gamma=100 \mathrm{~J} / \mathrm{m}^{2}$ and $\mu=0$ (see text for details).

Figure 3. Load $(P)$ - top fibre displacement $(d)$ for a fibre diameter $D=4 \mu \mathrm{m}$ and friction coefficient $\mu=0$. This set of simulations complements those shown in Fig. 2 and allows determination of the critical displacement $d_{\mathrm{cr}}$ in case the value is above $6 \mu \mathrm{m}$ (maximum top fibre displacement imposed for simulations in Fig. 2).

Figure 4. Interfacial strength $(\tau)$ - critical displacement $\left(d_{\mathrm{cr}}\right)$ curves for different values of $\Gamma$. This representation results in an interfacial behaviour map showing two zones: Zone (1), at the left-hand side of each curve, shows a region governed by the cohesive interaction between the fibre and the matrix, and (2) at the right-hand side of each curve, where the frictional contact between the part of the debonded fibre and the matrix dominates. It can be seen that, for high values of $\tau$ a change on $\Gamma$ will not greatly affect the value of $d_{c r}$, while the effect is increased when decreasing $\tau$. Effect of including thermal stresses will be to move the represented curves slightly to the right. See Figure S6 in the Supplementary Material for more information.

Figure 5. Interface fracture strength $(\Gamma)$ - critical displacement $\left(d_{\mathrm{cr}}\right)$ for different values of $\tau$. As in Fig. 4, this representation results in an interfacial behaviour map showing two zones: Zone (1), at the left-hand side of each curve, indicates a region governed by the cohesive interaction between the fibre and the matrix, and (2) at the right-hand side of each curve, where the frictional contact between the part of the debonded fibre and the matrix dominates. It is worthy to note here that dependency between $\Gamma$ and $d_{\mathrm{cr}}$ for each value of $\tau$ is linear, being the slope reduced while increasing $\tau$. In other words, also as in Fig. 4, it can be seen how for high values of $\tau$, a change in $\Gamma$ is not translated into a remarkable variation of $d_{c r}$, 
while the effect is increased when $\tau$ is reduced. Effect of thermal stresses will be to slightly reduce the slopes of the $\Gamma-d_{\mathrm{cr}}$ curves, see Figure $\mathrm{S} 6$ in the Supplementary Material for more information.

Figure 6. Evolution of the crack length with the top fibre displacement for an interfacial fracture strength $\Gamma=100 \mathrm{~J} / \mathrm{m}^{2}, D=4 \mu \mathrm{m}$ and different values of interface toughness $\tau$. The results reveal that the crack length propagation rate stabilizes with a constant slope for each value of $\tau$.

Figure 7. Propagation velocity of the interfacial crack, $m$, leading to fibre debonding in terms of the fibres' diameter $D$ for different values of the interface toughness $\tau$ and at a constant value of interfacial fracture strength, $\Gamma=200 \mathrm{~J} / \mathrm{m}^{2}$. Superimposed are the linear fitting functions using Eq. (4). That for $\tau \geq 100 \mathrm{MPa}$, the curves $S$ vs. $D$ virtually overlaps. Additional figures are available in the Supplementary Materials.

Figure S1. Load $(P)$ - top fibre displacement $(d)$ for an interfacial strength $\tau=50 \mathrm{MPa}$. Closed symbols are for a Young's modulus of the matrix $E=300 \mathrm{GPa}$ and open symbols for $E=50$ $\mathrm{MPa}$. Comparing fibres with same diameter $D$ and same value of interface fracture toughness $\Gamma$, it can be seen how strong variations on the Young's modulus of the matrix result in almost unnoticeable changes in the $P-d$ curves.

Figure S2. Load $(P)$ - top fibre displacement $(d)$ for an interface fracture toughness $\Gamma=100$ $\mathrm{J} / \mathrm{m}^{2}$ and different combinations of interfacial strength $(\tau)$, friction coefficient $(\mu)$ and fibre diameter (D). Closed symbols are for $\mu=0$ and open symbols for $\mu=0.05$. (a) $\tau=50 \mathrm{MPa}$; (b) $\tau$ $=75 \mathrm{MPa}$; (c) $\tau=100 \mathrm{MPa}$; (d) $\tau=200 \mathrm{MPa}$; (e) $\tau=300 \mathrm{MPa}$; (f) $\tau=500 \mathrm{MPa}$.

Figure S3. Load $(P)$ - top fibre displacement $(d)$ for an interface fracture toughness $\Gamma=150$ $\mathrm{J} / \mathrm{m}^{2}$ and different combinations of interfacial strength $(\tau)$, friction coefficient $(\mu)$ and fibre diameter (D). Closed symbols are for $\mu=0$ and open symbols for $\mu=0.05$. (a) $\tau=50 \mathrm{MPa}$; (b) $\tau$ $=75 \mathrm{MPa}$; (c) $\tau=100 \mathrm{MPa}$; (d) $\tau=200 \mathrm{MPa}$; (e) $\tau=300 \mathrm{MPa}$; (f) $\tau=500 \mathrm{MPa}$.

Figure S4. Load $(P)$ - top fibre displacement $(d)$ for an interface fracture toughness $\Gamma=200$ $\mathrm{J} / \mathrm{m}^{2}$ and different combinations of interfacial strength $(\tau)$, friction coefficient $(\mu)$ and fibre diameter (D). Closed symbols are for $\mu=0$ and open symbols for $\mu=0.05$. (a) $\tau=50 \mathrm{MPa}$; (b) $\tau$ $=75 \mathrm{MPa}$; (c) $\tau=100 \mathrm{MPa}$; (d) $\tau=200 \mathrm{MPa}$; (e) $\tau=300 \mathrm{MPa}$; (f) $\tau=500 \mathrm{MPa}$.

Figure S5. Load $(P)$ - top fibre displacement $(d)$ for an interface fracture toughness $\Gamma=250$ $\mathrm{J} / \mathrm{m}^{2}$ and different combinations of interfacial strength $(\tau)$, friction coefficient $(\mu)$ and fibre diameter $(D)$. Closed symbols are for $\mu=0$ and open symbols for $\mu=0.05$. (a) $\tau=50 \mathrm{MPa}$; (b) $\tau=75 \mathrm{MPa}$; (c) $\tau=100 \mathrm{MPa}$; (d) $\tau=200 \mathrm{MPa}$; (e) $\tau=300 \mathrm{MPa}$; (f) $\tau=500 \mathrm{MPa}$.

Figure S6. Load $(P)$ - top fibre displacement $(d)$ comparing the situations of a relaxed system (no residual stresses) with a system presenting residual stresses originated from a cooling down process $(\Delta T=100 \mathrm{~K})$. Here, four extreme combinations for the interface properties have been considered with $\tau=50$ and $500 \mathrm{MPa}$ and $\Gamma=100$ and $250 \mathrm{~J} / \mathrm{m}^{2}$ in order to maximize the effects that residual stresses may have. Part (a) is for a fibre's diameter $D=4 \mu \mathrm{m}$ while part (b) is for $D=9 \mu \mathrm{m}$. As it can be observed, thermal stresses do not affect the overall morphology of 
the $P-d$ curves, though the onset of the frictional governed regime is slightly delayed. This will imply that curves in Fig. 4 will move to the right, while in Fig. 5 the slopes will be reduced due to thermal stress.

Figure S7. Experimental quasistatic load $(P)$ - top fibre displacement $(d)$ curves for two different fibre's diameters $(D)$. Solid black line is for $D \sim 7 \mu \mathrm{m}$ and dashed red line for $D \sim 5$ $\mu \mathrm{m}$. Same tendencies as in the simulations can be observed, where higher diameters require of higher loads to impose the same displacement.

Figure S8. Fibre debonding - interfacial strength $(\tau)$ plot showing the initial debonding after cooling down and previously to push-in. Variation in temperature corresponds to $\Delta \mathrm{T}=100 \mathrm{~K}$ and initial debonding is independent of the interface fracture toughness $\Gamma$. For low values of $\tau$ initial debonding is more important for bigger fibres (here open symbols are for $D=4 \mu \mathrm{m}$ and close symbols for $D=9 \mu \mathrm{m}$ ) but, as $t$ is increased, difference is reduced and for higher enough $\tau$ initial debonding disappears. Hence, the effect of having different values of coefficient of thermal expansion for the fibre and the matrix is reduced when having high values of interfacial strength. Note that for $\tau=500 \mathrm{MPa}$ results overlap.

Figure S9. Propagation speed of the interfacial crack $m$ leading to fibre debonding in terms of the fibres' diameter $D$ for different values of the interface toughness $\tau$. Closed symbols are for $\mu=0$ and open symbols for $\mu=0.05$. (a) $\Gamma=100 \mathrm{~J} / \mathrm{m}^{2}$; (b) $\Gamma=150 \mathrm{~J} / \mathrm{m}^{2}$; (c) $\Gamma=200 \mathrm{~J} / \mathrm{m}^{2}$; (d) $\Gamma=250 \mathrm{~J} / \mathrm{m}^{2}$. Notice how for part (a) $S$ is almost independent on $\tau$. As $\Gamma$ increases, $m$ becomes more sensitive to $\tau$ for a given value of $D$. 


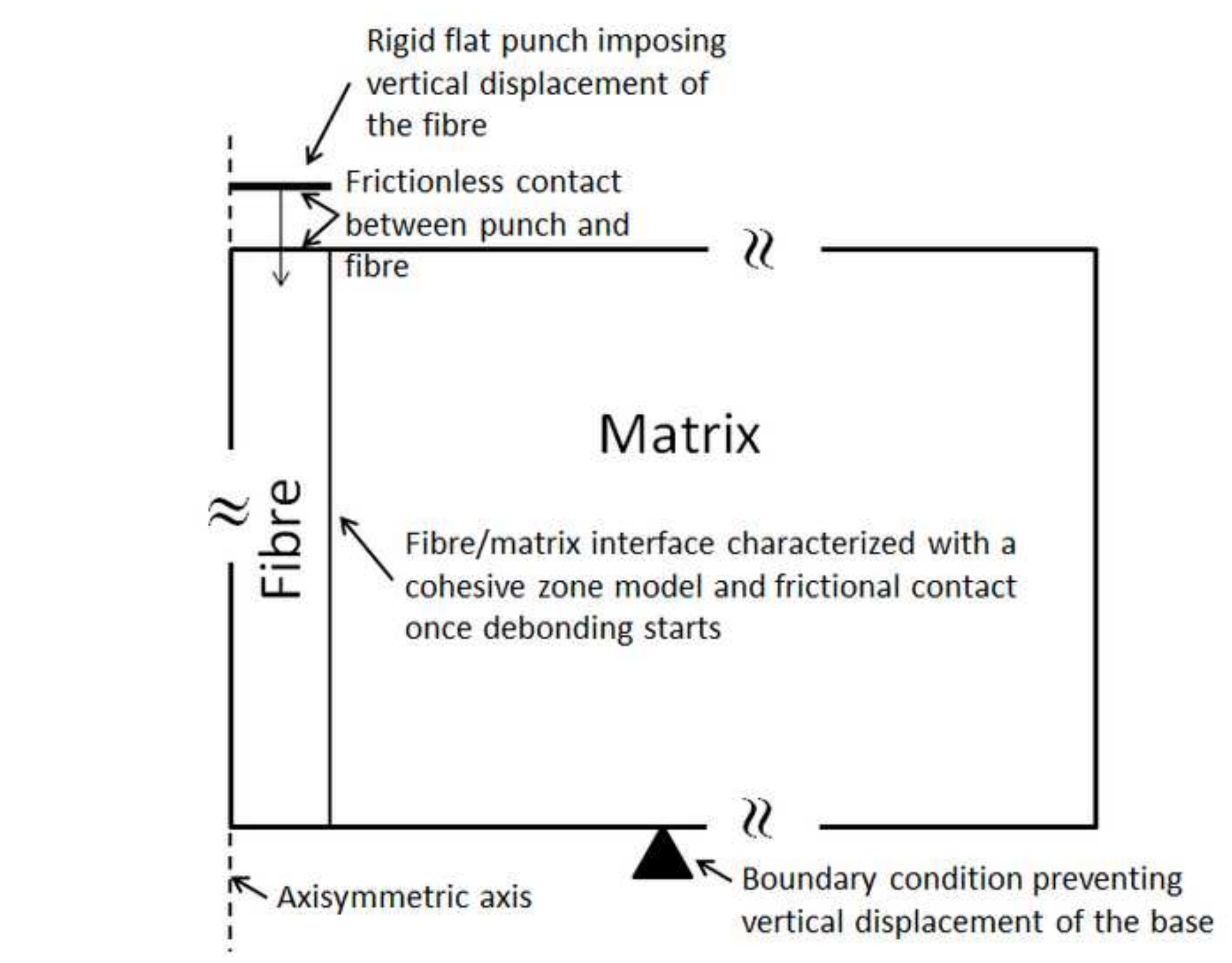

Rigid flat punch imposing

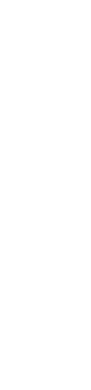

.


Figure 2

Click here to download high resolution image

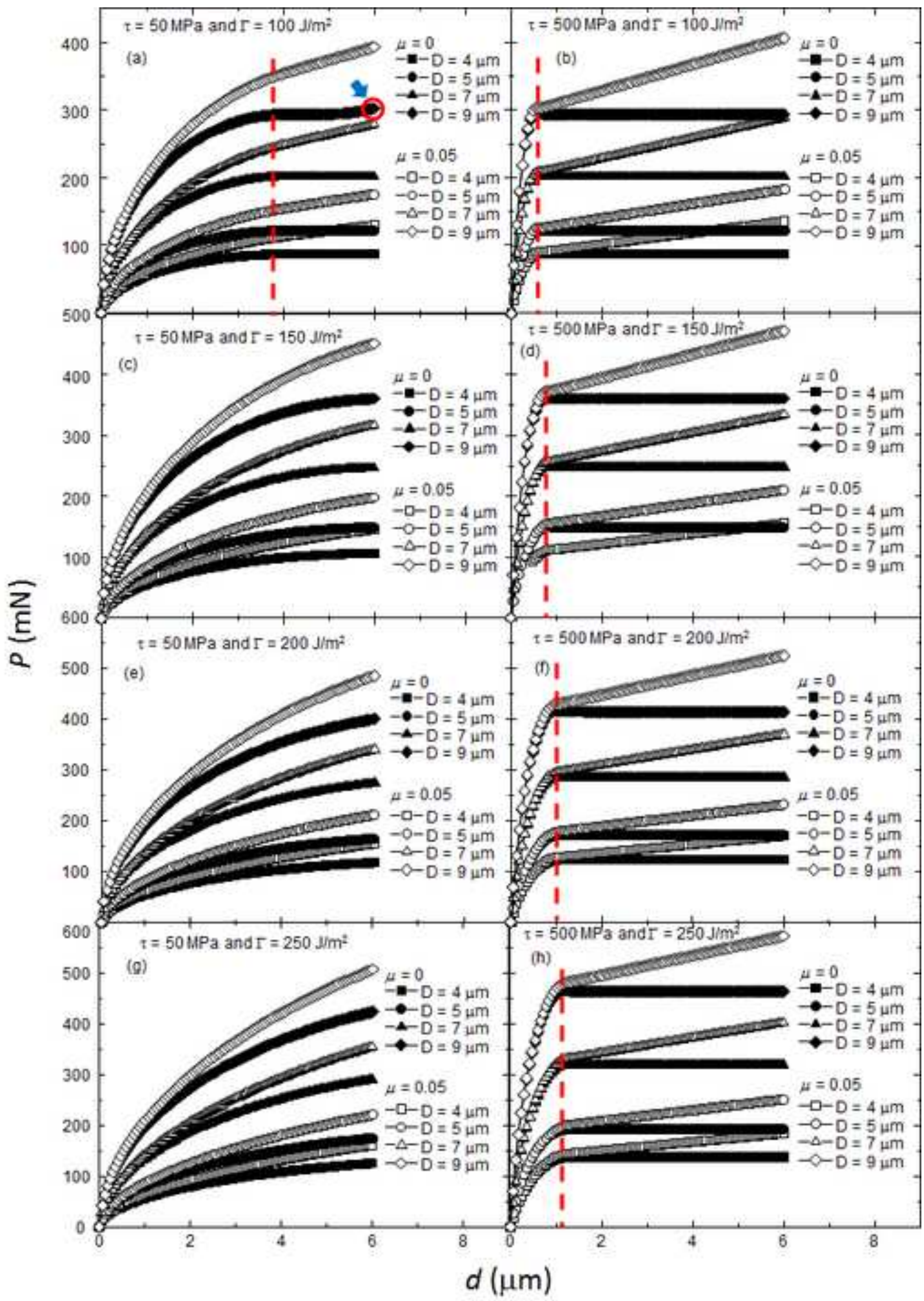




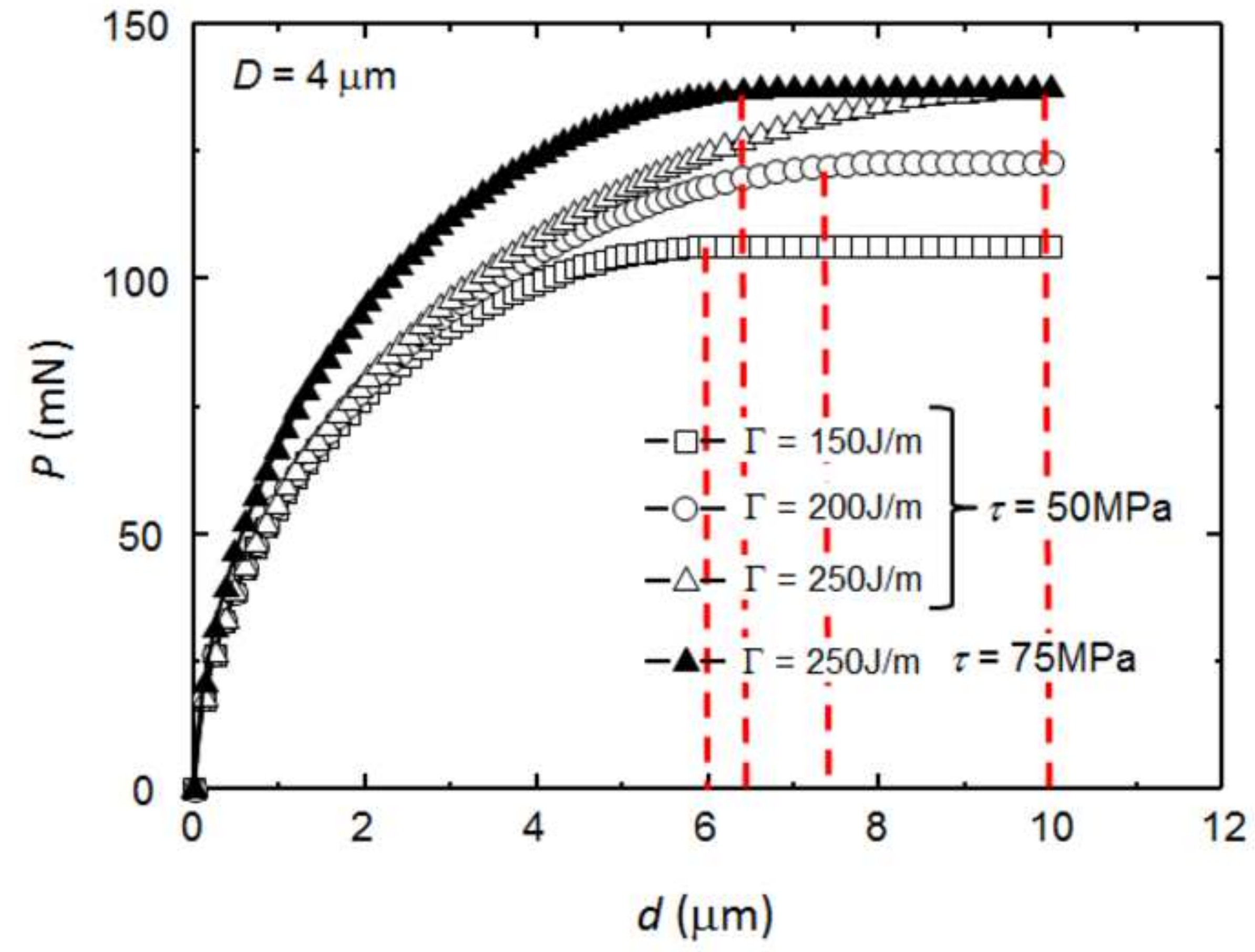




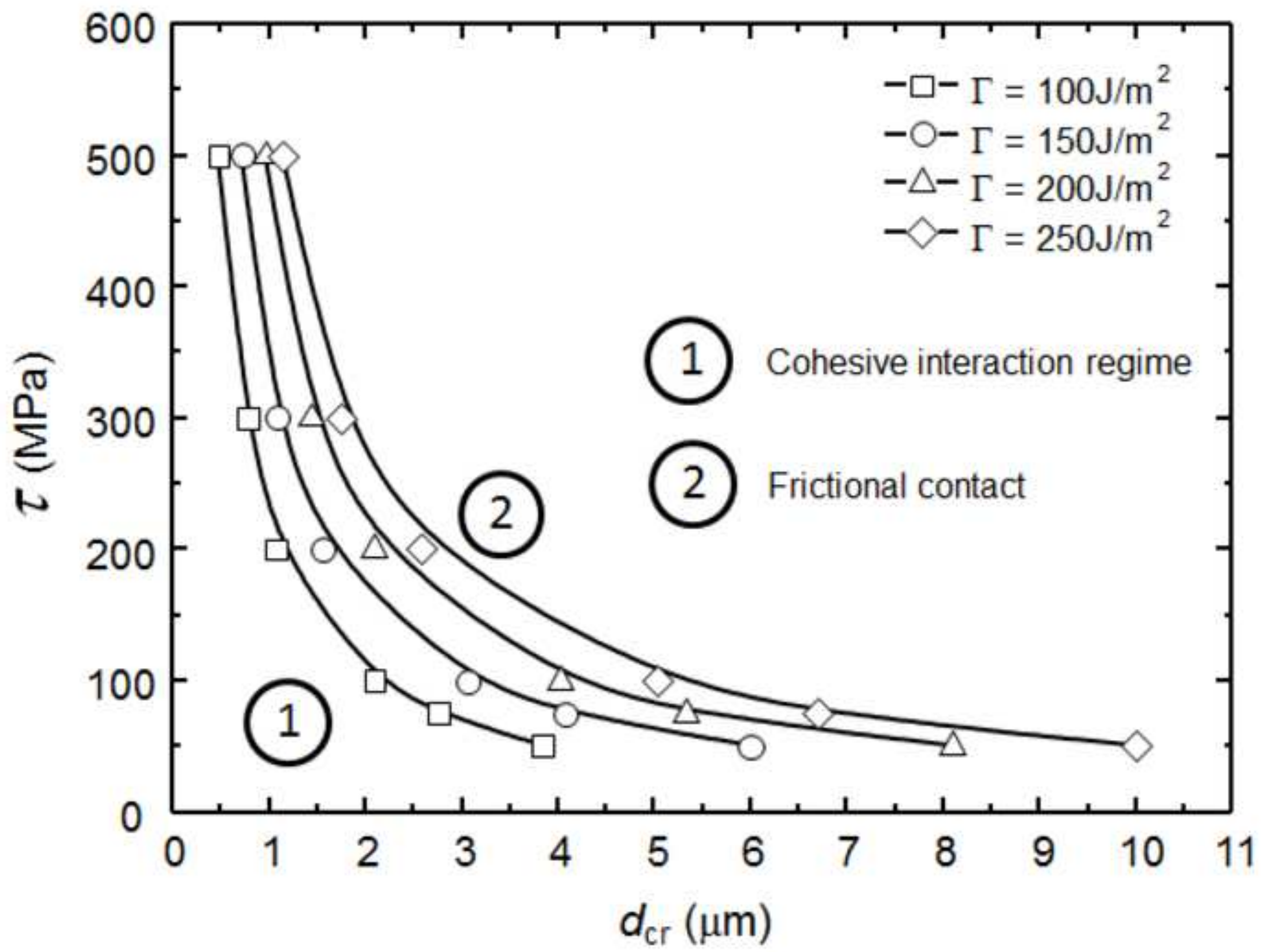




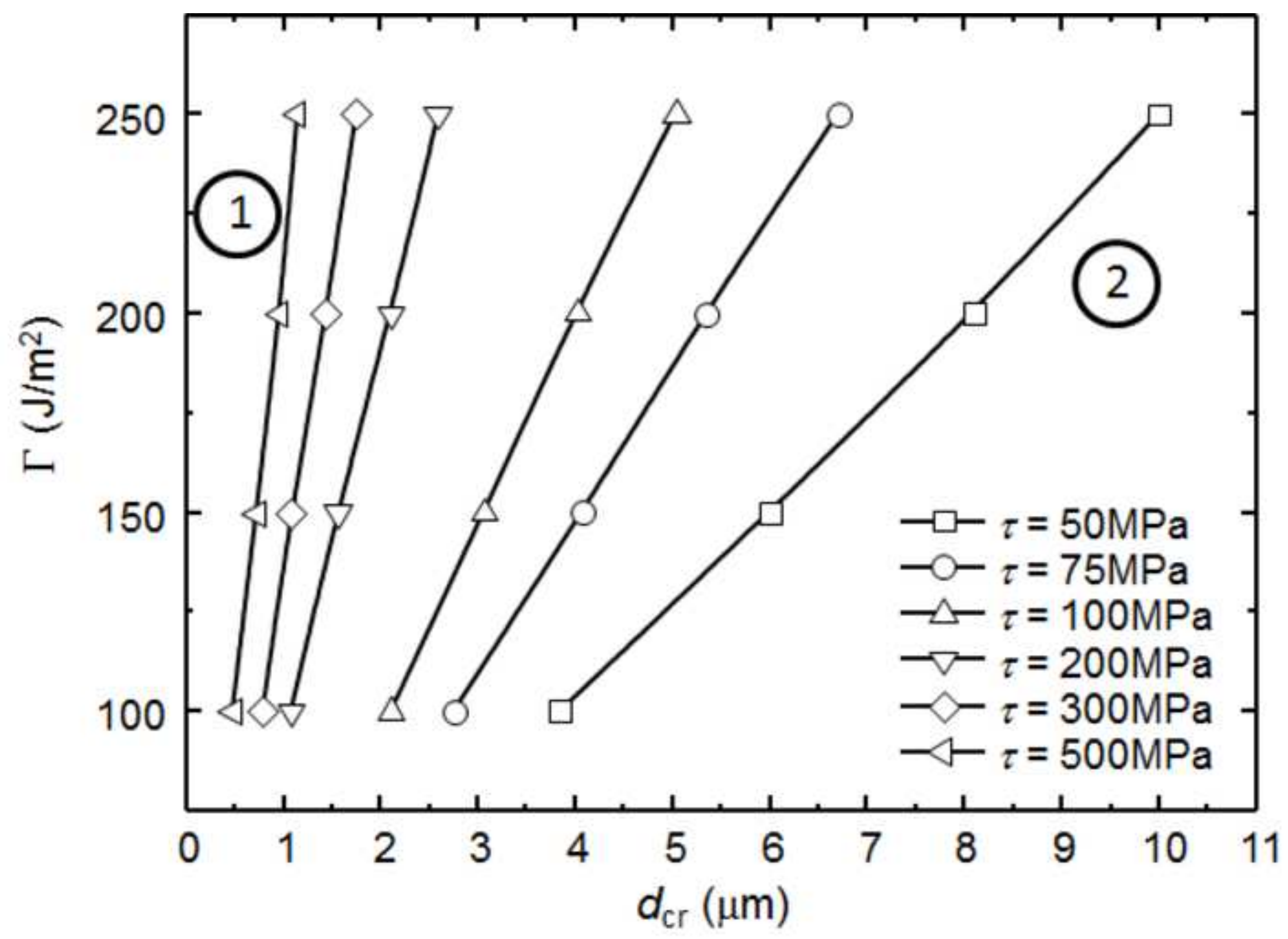




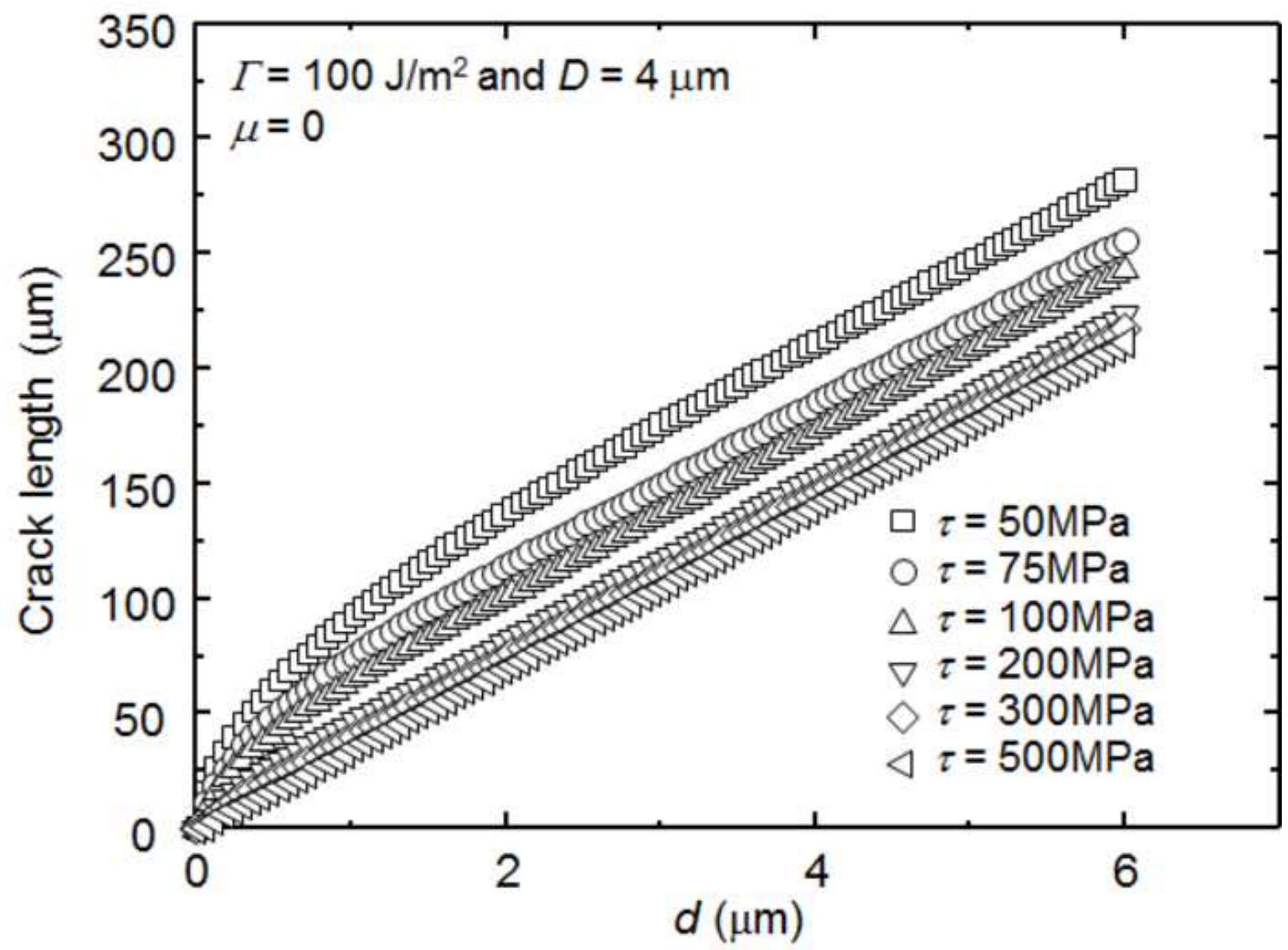




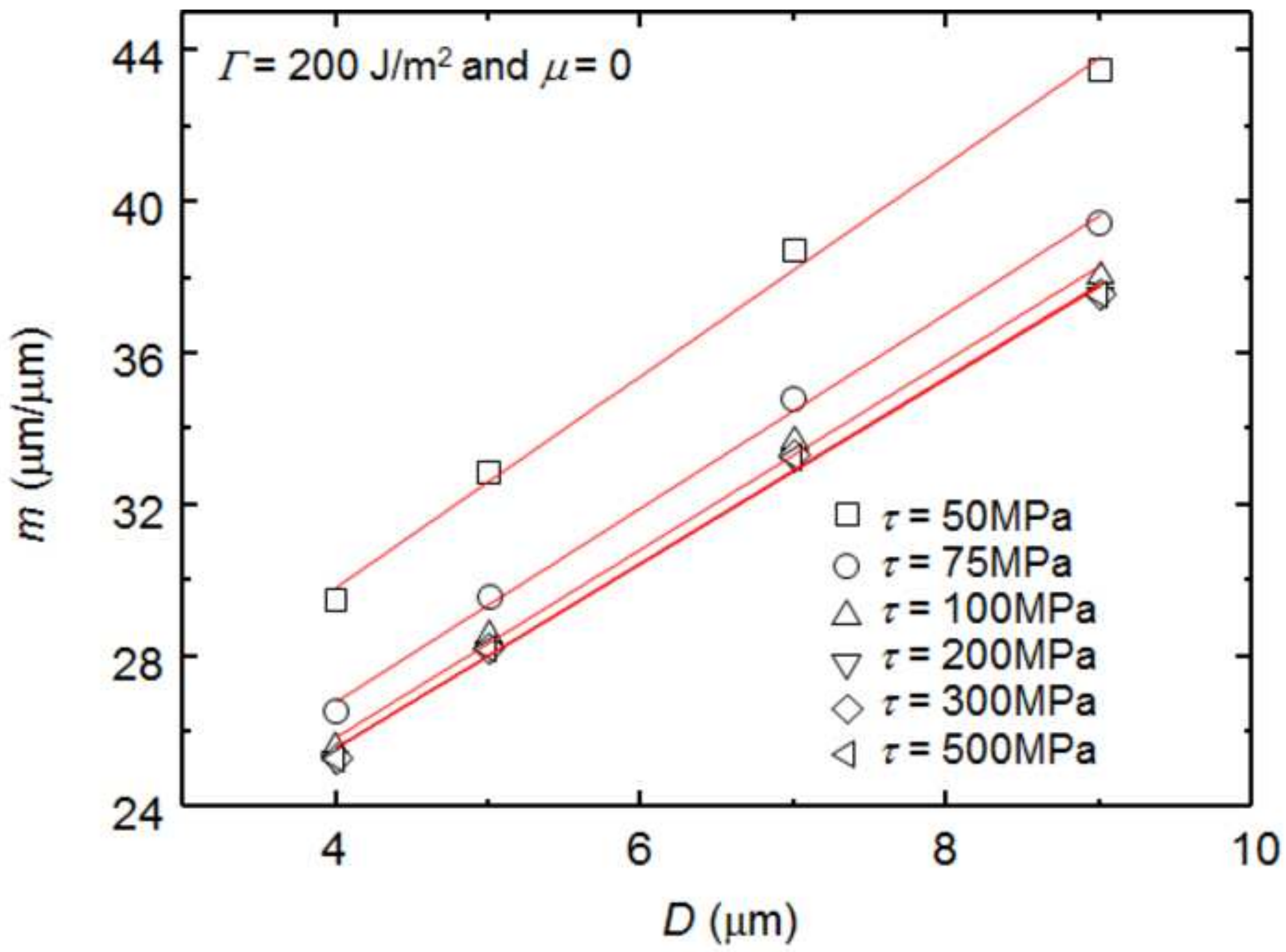

